\title{
The association between SARS-CoV-2 RT-PCR cycle threshold and mortality in a community cohort
}

\begin{abstract}
To the Editor:
Copyright (CThe authors 2021.

This version is distributed under the terms of the Creative Commons Attribution Non-Commercial Licence 4.0. For commercial reproduction rights and permissions contact permissions@ersnet.org

Received: 5 Feb 2021 Accepted: 2 June 2021

\section{○@®@}

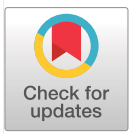

A coronavirus disease 2019 (COVID-19) diagnosis is widely made by the use of reverse transcription polymerase chain reaction (RT-PCR) testing for severe acute respiratory syndrome coronavirus 2 (SARS-CoV-2). When using RT-PCR, the infectivity of SARS-CoV-2 can be inferred from the threshold cycle (Ct) value [1]. It is best practice to confirm the validity of the standard curve using reference materials or in-house plasmid controls with known viral copy numbers [2]. As the Ct value represents the cycle number at which the signal breaches the threshold for positivity, a lower Ct value is indicative of a higher viral load. Although some studies suggest that viral load is associated with mortality and infectiousness [3, 4], a systematic review has identified little difference in viral load between pre-symptomatic, asymptomatic and symptomatic patients [5]. As a result, the clinical relevance of viral load remains controversial, and it is not used in clinical practice [6]. Here, we report the relationship between the $\mathrm{Ct}$ value and all-cause mortality for people who tested positive for SARS-CoV-2 on a combined nasal and pharyngeal swab in the Tayside region of Scotland, UK. This is a community cohort study and includes the local population of the region, as well as symptomatic health and social care workers tested as part of a screening programme [7]. In order to obtain clinical characteristics and outcomes for those who tested positive, anonymised record linkage was conducted between routine healthcare datasets as described previously [8]. All positive PCR tests from 12 March until 1 May, 2020 were included, and all deaths recorded by National Records Scotland until 20 May, 2020. Approval for anonymised data linkage was granted by the local Data Protection Officer (Caldicott Guardian).

A total of 1337 individuals testing positive for SARS-CoV-2 were recorded in NHS Tayside. In the laboratory, nucleic acid was extracted and a one-step RT-PCR assay targeting the RdRP gene using an ABI7500 as previously described. For individuals with multiple positive tests, the Ct value from the first of these was included, and for multiple tests on the same day, the test with the lowest Ct value was used. In our cohort, the overall median (interquartile range) Ct value of an individual's first positive test was 28.7 (23.9-33.4). The median (interquartile range) age of the cohort was 53 years (40-72 years), and 61.9\% were female. 202 individuals (15\%) were hospitalised with COVID-19 (recorded with the ICD code for COVID-19: U07.1).

The total number of deaths in the cohort was 161 (12\%), the majority of deaths (102) occurred in a non-hospital cohort. However, a larger proportion of those hospitalised died (29\% compared to $9 \%$ who died without hospitalisation). This is likely due to the high incidence of infection and deaths in care homes for the elderly. Mean \pm SD follow-up available was $37 \pm 14$ days, and maximum follow up was 69 days. $15 \%$ of the cohort had hypertension, $4 \%$ had heart failure, $9 \%$ had ischaemic heart disease, $8 \%$ had COPD, 5\% had asthma, $8 \%$ had chronic kidney disease and 10\% had type 2 diabetes mellitus.

A Cox proportional hazards model adjusting for relevant clinical factors was used to test the association between Ct value and mortality. These were: age, sex, hypertension, heart failure, ischaemic heart disease, COPD, asthma, chronic kidney disease and type 2 diabetes mellitus. The model showed a significant association between initial Ct value and mortality (HR 0.96, 95\% CI 0.93-0.99; p=0.0044). Other independent predictors of mortality were age (HR 1.08, 95\% CI 1.06-1.09; $\mathrm{p}<0.001$ ), and male sex (HR 1.77, 95\% CI 1.28-2.44; $\mathrm{p}<0.001)$.

Shareable abstract (@ERSpublications)

Ct values from RT-PCR tests are associated with risk of mortality in SARS-CoV-2 infection. Hazards of $\mathrm{Ct}$ values $<20$ compared to $>30$ were $2.20(95 \% \mathrm{Cl} 1.28-3.76)$ in a model adjusted for age, sex, comorbidities and hospitalisation. https://bit.ly/3gjuqdU

Cite this article as: Waudby-West R, Parcell BJ, Palmer CNA, et al. The association between SARS-CoV-2 RT-PCR cycle threshold and mortality in a community cohort. Eur Respir J 2021; 58: 2100360 [DOI: 10.1183/13993003.00360-2021].
\end{abstract}




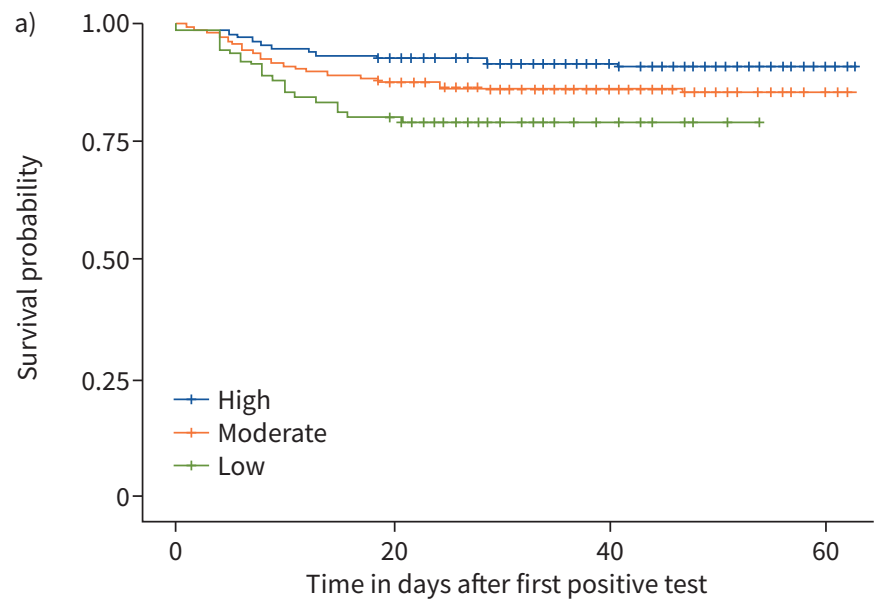

Number at risk

High $\quad 563$

Moderate 683

Low 91

$\begin{array}{cc}520 & 316 \\ 597 & 336 \\ 73 & 17\end{array}$

b)

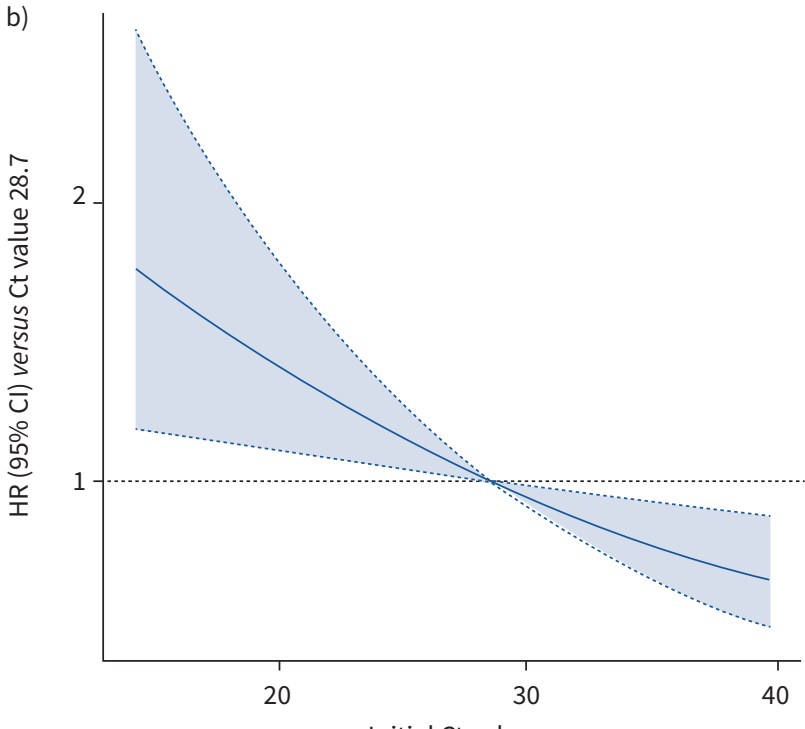

FIGURE 1 a) Overall survival probability for individuals positive for SARS-CoV-2 in Tayside, Scotland based on the cycle threshold (Ct) value of their first positive test. Crosses represent censored individuals. b) Estimated hazard ratio (HR) based on initial Ct value of individual in a model adjusting for age, sex, hypertension, heart failure, ischaemic heart disease, COPD, asthma, chronic kidney disease and type 2 diabetes mellitus.

Ct values were then stratified into high (>30), moderate (20-30), and low $(<20)$, and a model was constructed adjusting for the same clinical factors (figure 1). This showed that a low initial Ct value was associated with increased hazard of mortality compared to a high initial Ct value (HR 2.10, 95\% CI 1.233.60; $\mathrm{p}=0.0069$ ); however, the same relationship between moderate and high was not statistically significant (HR 1.37, 95\% CI 0.96-1.94; p=0.08). We performed a sensitivity analysis adjusting for hospitalisation status as a marker of disease severity; in this adjusted analysis, a low initial Ct value increased hazards of mortality by 2.20 (95\% CI 1.28-3.76; $\mathrm{p}=0.0005)$. These results suggest that disease severity does not confound the association between $\mathrm{Ct}$ values and mortality.

Early identification of the risk that COVID-19 presents to an individual remains a challenge. Most clinical investigations for COVID-19 disease have been limited to hospitalised cohorts; here, we were able to assess the value of Ct scores in the community. However, as this is a community cohort study, data from clinical investigations performed on hospitalised patients that help classify disease severity were not available for the entire cohort. Here, we have demonstrated an independent relationship between the Ct value (indicative of viral load) of an individual's first positive SARS-CoV-2 PCR test, and overall mortality, in which a lower Ct value is associated with greater hazards of death. Ct values may allow for early risk stratification in patients with COVID-19. Further work is required to confirm this association and to assess the utility of including $\mathrm{Ct}$ values in early risk stratification.

Rupert Waudby-West ${ }^{1}$, Benjamin J. Parcell ${ }^{2}$, Colin N.A. Palmer ${ }^{1}$, Samira Bell $\oplus^{1}$, James D. Chalmers ${ }^{3}$ and Moneeza K. Siddiqui $\oplus^{1}$

${ }^{1}$ Division of Population Health and Genomics, School of Medicine, University of Dundee, Dundee, UK. ${ }^{2}$ Dept of Medical Microbiology, Ninewells Hospital and Medical School, Dundee, UK. ${ }^{3}$ Division of Molecular and Clinical Medicine, School of Medicine, University of Dundee, Dundee, UK.

Corresponding author: James D. Chalmers (j.chalmers@dundee.ac.uk)

Conflict of interest: R. Waudby-West has nothing to disclose. B.J. Parcell has nothing to disclose. C.N.A. Palmer has nothing to disclose. S. Bell has nothing to disclose. J.D. Chalmers has received research grants from GlaxoSmithKline, Boehringer Ingelheim, AstraZeneca, Pfizer, Grifols, Bayer AG, Polyphor and Insmed; and received consultancy, congress travel or speaker fees from GlaxoSmithKline, Bayer Healthcare, Aradigm Corporation, Grifols, Pfizer, Boehringer Ingelheim, Napp and Insmed. M.K. Siddiqui has nothing to disclose. 


\section{References}

1 Public Health England. Understanding Cycle Threshold (Ct) in SARS-CoV-2 RT-PCR. London, Public Health England, 2020. Available from: https://assets.publishing.service.gov.uk/government/uploads/system/uploads/ attachment_data/file/926410/Understanding_Cycle_Threshold__Ct_in_SARS-CoV-2_RT-PCR_.pdf

2 Han MS, Byun JH, Cho Y, et al. RT-PCR for SARS-CoV-2: quantitative versus qualitative. Lancet Infect Dis 2020; 21: 165.

3 Pujadas E, Chaudhry F, McBride R, et al. SARS-CoV-2 viral load predicts COVID-19 mortality. Lancet Respir Med 2020; 8: e70.

4 Singanayagam A, Patel M, Charlett A, et al. Duration of infectiousness and correlation with RT-PCR cycle threshold values in cases of COVID-19, England, January to May 2020. Euro Surveill 2020; 25: 2001483.

5 Walsh KA, Jordan K, Clyne B, et al. SARS-CoV-2 detection, viral load and infectivity over the course of an infection. J Infect 2020; 81: 357-371.

6 Kelleni MT. SARS-CoV-2 viral load might not be the right predictor of COVID-19 mortality. J Infect 2020; 82: E35.

7 Parcell BJ, Brechin K, Allstaff S, et al. Drive-through testing for SARS-CoV-2 in symptomatic health and social care workers and household members: an observational cohort study. Thorax 2020; 75: 1109-1111.

8 Siddiqui MK, Parcell BJ, Allstaff S, et al. Characteristics and outcomes of health and social care workers testing positive for SARS-CoV-2 in the Tayside region of Scotland. Eur Respir J 2020; 56: 2002568. 